\title{
EL ABORDAJE DE LA EDUCACIÓN SEXUAL EN LAS FAMILIAS DE LOS ESTUDIANTES DE LAS INSTITUCIONES EDUCATIVAS DE BÁSICAY Y MEDIA OFICIALES DEL MUNICIPIO DE ARMENIA, QUINDIO, Y SU INFLUENCIA EN LA DINÁMICA FAMILIAR
}

\author{
AN APPROACH OF SEXUAL EDUCATION FROM STUDENT'S FAMILIES FROM BASIC \\ AND MEDIA OFFICIAL EDUCATIONAL OF THE MUNICIPALITY OF \\ ARMENIA, QUINDÍO, AND ITS INFLUENCE IN FAMILY DYNAMIC
}

Jhon Jaime De La Rosa B. ${ }^{1}$; Carmen Aydé Fernández R. ${ }^{2}$; Mónica Soto R. ${ }^{3}$

1. Facultad de Ciencias Humanas y Bellas Artes, Programa de Trabajo Social

2 Facultad de Ciencias de la Salud, Programa de Enfermería

3 Secretaria de Educación Municipal de Armenia

Integrantes de grupo de investigación En Desarrollo de la Universidad del Quindío

Recibido: 10 de Septiembre de 2014

Aceptado: 30 de Noviembre de 2014

Correspondencia del autor: E-mail jjdelarosa@uniquindio.edu.co

\section{RESUMEN}

Este artículo surgió de la investigación cualitativa "El abordaje de la educación sexual en las familias de los estudiantes de las instituciones educativas de básica y media oficiales del municipio de Armenia, Quindío, y su influencia en la dinámica familiar", financiado por la Vicerrectoría de Investigaciones de la Universidad del Quindío, en donde se analizaron las variaciones de las funciones de la sexualidad de los niños, niñas y adolescentes (NNA), dentro de la dinámica familiar. Para la recolección de información se realizaron entrevistas a los diferentes actores de las instituciones educativas y a las familias de los estudiantes. Así mismo se consolidaron categorías inductivas que demostraron que el abordaje de la educación sexual en las familias se circunscribe al control de la natalidad, a las infecciones de transmisión sexual (ITS) y al deseo de tener una pareja estable; además, se reveló un gran temor frente al tema de la sexualidad por la deficiente formación y un inadecuado acompañamiento de la familia de origen. Las características de los entrevistados del núcleo familiar fueron: un promedio de edad de 40 años, estudios secundarios, con procedencia en su mayoría de los departamentos de Caldas, Valle del Cauca y Putumayo, viven hace 4 años en la ciudad, son empleados y poseen una tipología familiar reconstituida y monoparental. Las variaciones en la dinámica familiar son, en su mayoría, establecimiento de normas y reglas al interior del hogar, compartidas o consensuadas, utilización del diálogo para la solución de problemas y vínculos familiares fortalecidos a través de los espacios compartidos durante el tiempo libre, las expresiones de afecto y el apoyo familiar.

Palabras clave: Familia, Sexualidad, Educación Sexual, Dinámica familiar. 


\begin{abstract}
This article emerged from the qualitative research "The approach to sex education in families of students of educational institutions of basic and official media in Armenia, Quindio, and their influence on family dynamics", funded by the Vice Presidency of Research at the University of Quindio, where changes in the functions of the sexuality of children and adolescents (NNA) within family dynamics were analyzed. For information gathering interviews with the different actors of the educational institutions and students' families were made. Also inductive categories that showed that the approach to sex education in families is limited to birth control, and sexually transmitted infections (STIs) and the desire to have a partner were consolidated; also great fear was revealed on the issue of sexuality by poor training and inadequate support from family of origin. The characteristics of the household respondents were: an average age of 40 years, secondary education, with provenance mainly from the departments of Caldas, Valle del Cauca and Putumayo, living four years ago in the city, are employed and have a reconstituted and single parent family typology. Changes in family dynamics are, mostly, setting norms and rules within the household, shared or consensual, using dialogue to solve problems and family ties strengthened through shared during leisure spaces, expressions of affection and family support.
\end{abstract}

Keywords: Family, Sexuality, Sexual Education, Family Dynamic.

\section{INTRODUCCIÓN}

La investigación denominada "El abordaje de la educación sexual en las familias de los estudiantes de las instituciones educativas IE de básica y media oficiales del municipio de Armenia, Quindío, y su influencia en la dinámica familiar" y realizada durante los años 2013 y 2014, tuvo como objetivo analizar la realidad social de las familias, asumidas como padres, madres o acudientes, de los estudiantes de los mencionados establecimientos educativos, en términos del abordaje de la educación sexual. El estudio se inscribió en la línea de investigación Formas de Cohesión Social, del programa Trabajo Social y al grupo de investigación En Desarrollo de la Universidad del Quindío, clasificado en la categoría $\mathrm{C}$ de Colciencias, el cual explora dimensiones del currículo, asociadas a las consideraciones epistemológicas, teóricas y metodológicas de la carrera de Trabajo Social.

Para el análisis de la problemática en el contexto local, se seleccionó como línea de base el estudio titulado "La educación sexual como tema transversal en la básica primaria de las instituciones educativas del municipio de Armenia, Quindío" (Fernández Rincón \& López Cardozo, 2011), según el cual la participación de las familias de los estudiantes en el desarrollo de la educación sexual es nula. De igual forma, se tuvo en cuenta el Plan Nacional de Educación Sexual (PNES), en donde se estipula que el abordaje de la transversalidad es inadecuado y los componentes teóricos que soportan el proyecto están basados en la función reproductiva de la familia.

De otra parte, se tomó como referencia la investigación "Nuevas configuraciones de las familias en los hogares de los estudiantes de las instituciones educativas de básica y media oficiales del municipio de Armenia Quindío, 2010" (De La Rosa Bobadilla, y otros, 2010), en donde se sugiere que las variaciones en la dinámica familiar se dan a partir del ejercicio de las funciones de la sexualidad de los NNA, desde la perspectiva de la representación de la autoridad, la comunicación y las relaciones afectivas. Igualmente, el estudio arroja los siguientes consolidados: el $83 \%$ de los hogares está compuesto por tres integrantes; existe un predominio de tipologías familiares nucleares completas y extensas, alrededor del 35\% y 33\% respectivamente; y acerca de la estructura familiar, en el $44.8 \%$ de las familias se reconoce la existencia de un jefe de hogar que ejerce como función fundamental la protección, mientras que el 34\% reconoce la presencia de un esposo, cuya función primordial es la de proveedor económico.

\section{ASPECTOS TEÓRICOS}

En el ámbito conceptual, son pilares fundamentales en la investigación los términos sexualidad, educación sexual (ES) y dinámica familiar, sobre los cuales es conveniente explorar algunos de sus tópicos teóricos. 
En primer lugar, la sexualidad es un aspecto central del ser humano presente a lo largo de toda su vida; contempla el sexo, las identidades y los papeles de género, la orientación sexual, el erotismo, el placer, la intimidad y la reproducción. Se vivencia y se expresa a través de pensamientos, fantasías, deseos, creencias, actitudes, valores, conductas, prácticas, papeles y relaciones interpersonales, y aunque incluye todas estas dimensiones, no todas se vivencian o se expresan de la misma manera (Organización Panamericana de la Salud, 1995). Adicionalmente plantea Weeks (1998) que dicho aspecto existe como una presencia social palpable, que configura nuestra figura pública y personal. Pero aclara que lo que se define como sexualidad es una construcción histórica, que reúne una multitud de distintas posibilidades biológicas y mentales, como la identidad genérica, la diferencias corporales, las capacidades reproductivas, las necesidades, los deseos y las fantasías; elementos que no necesariamente deben estar vinculados, y que en otras culturas no lo han estado. Así mismo, hoy en día "el sexo" tiene un significado dominante y usual, desde principios del siglo XIX, el cual se refiere a las relaciones físicas entre los sexos, es decir, a "tener sexo".

En el mismo tenor, el Ministerio de Educación Nacional / Fondo de Población de las Naciones Unidas, UNFPA, (2008), conceptúa que "la sexualidad es una abstracción. La sexualidad es, ante todo, una construcción mental de aquellos aspectos de la existencia humana que adquieren significado sexual y por lo tanto, nunca es un concepto acabado y definitivo pues la exigencia misma es continua y cambiante". Sobre el particular Marina (2005) llama sexualidad al "universo simbólico construido sobre una realidad biológica: el sexo". En general un alto número de enfoques teóricos parten de la característica biológica, pero se distancian en un amplio abanico de autores, en donde algunos le atribuyen a "la sexualidad un imperativo biológico que, ante la estructura social y educativa, lucha por expresarse"; otros, como Rubio (1994), ven la sexualidad básicamente como "la resultante de la interacción grupal que, a partir de una base biológica relativamente invariante, origina diversidad de características de ideas, sentimientos, actitudes, entre otros, esto se evidencia a través de la regulación social e institucional de lo que el grupo entiende por sexualidad".

Se devela también que la sexualidad es una construcción simbólica de realidades propias, donde convergen experiencias sociales y culturales, y vivencias colectivas, a través de las cuales nos expresamos como seres sexuados, en una sociedad determinada. Por consiguiente, la sexualidad se convierte en un factor constitutivo del ser humano, desde las dimensiones que la nutren como lo biológico, lo psicológico, lo socio cultural, y lo ético. Comprende además aspectos emocionales, comportamentales, cognitivos y comunicativo, tanto para el desarrollo de lo individual como de lo social. Sobre esto último, la sexualidad

Cuadro 1: Dimensiones de la sexualidad

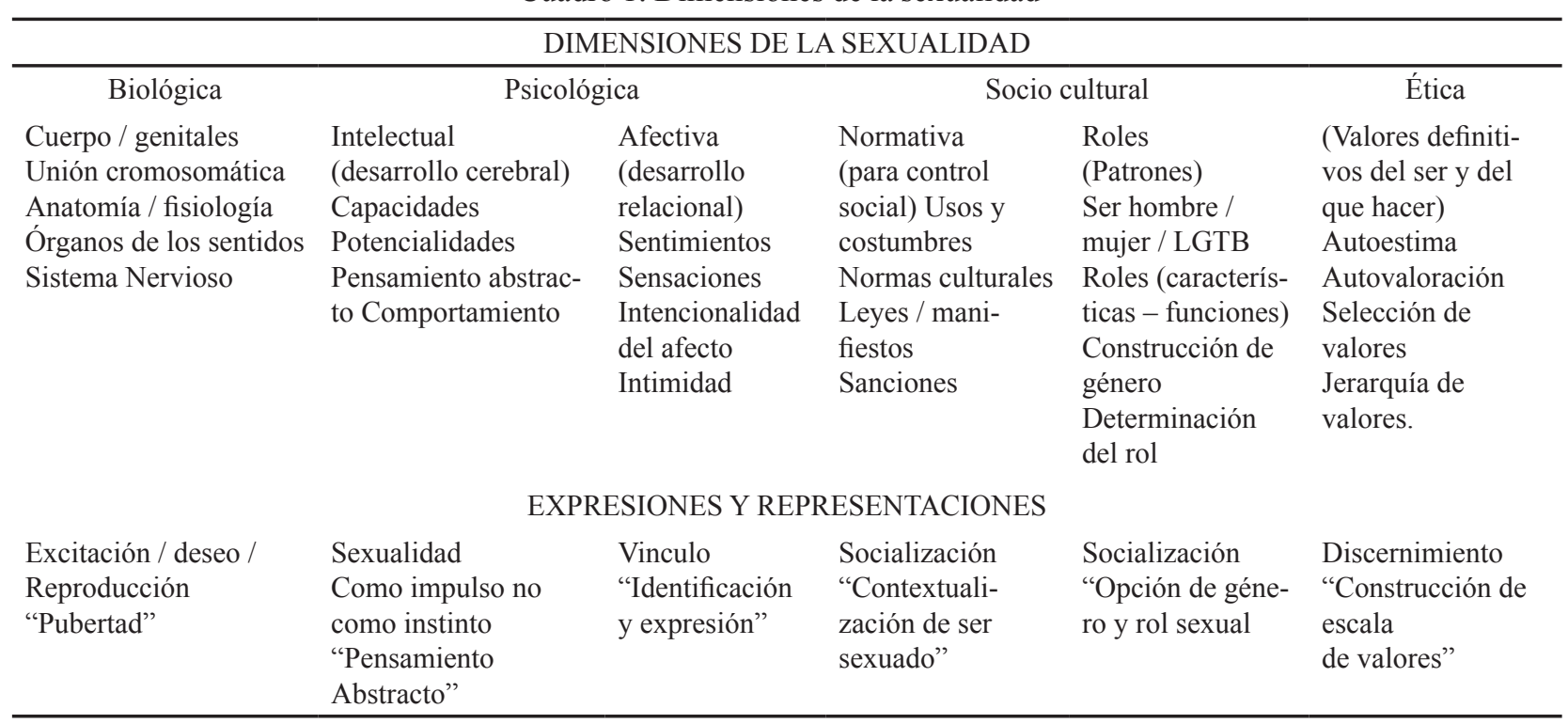

Fuente: Elaborado por los autores. 
En segundo lugar, la educación sexual (ES) se interpreta como un proceso de preparación de las personas a lo largo de su vida para el encuentro libre, responsable y pleno de su sexualidad con la de los demás, por supuesto, desde sus propias dimensiones y funciones. Este escenario se debe potenciar por la apropiación de experiencias, emociones y conocimientos, evidenciados posteriormente en habilidades, actitudes, valores y prácticas que favorecen el ejercicio pleno de la propia sexualidad, al decir de Velandia (citado por Arreguez, 2013).

Siguiendo estos postulados, la ES debe potenciar la construcción de las subjetividades, en la línea de posibilitar la identificación de las propias capacidades, la aceptación de derechos y deberes y la preparación emocional, como condición para asumir los procesos particulares, relacionales y sociales que se articulan en la sexualidad. En opinión de Cardinal (1975) se debe permitir el reconocimiento, la aceptación y la valoración del cuerpo; la necesidad de cuidarlo, posibilita el aprendizaje de su estructura, cambios y funcionamiento; así mismo facilita el desarrollo de sentimientos de autoestima y seguridad. Por supuesto, lo anterior conlleva a la necesidad de desarrollar procesos de toma de decisiones, uso adecuado del tiempo libre, el reconocimiento de los roles sexuales, la necesidad de una perspectiva de género, la importancia del eros, la inclusión de la intimidad, la incorporación del amor en la sexualidad, y por añadidura la adquisición de valores como la libertad, la responsabilidad, la tolerancia, la igualdad, la fraternidad, la paz y la vida. Así pues, la educación sexual debe ser: "una educación para el ser más que para el hacer, para la formación de autoconciencia, para el cambio y para la libertad" (Cardinal, 1975).

El tercer concepto básico de la investigación se relaciona con la dinámica familiar, aspecto que se define bajo las nociones de estructura y composición familiar, tipos de familia, relaciones familiares, funciones de la familia, comunicación, reglas, normas y valores. En este horizonte se cuenta, por un lado, con el desarrollo de las dimensiones y funciones de la sexualidad de los NNA, situación que demanda cambios en el sistema familiar, debido a sus necesidades de desarro1lo que incorporan la independencia y la autonomía. Por otro lado, aparece la dinámica familiar que en su conjunto moldean los comportamientos de cada individuo; interinfluencia que puede explicar los diversos comportamientos de la familia frente al abordaje de la educación sexual.

Consecuente con lo anterior, la dinámica familiar se puede asumir como el proceso que se genera al interior de un grupo, el cual es interpretado como el manejo de interacciones y relaciones de los miembros de la familia, quienes estructuran una determinada organización, estableciendo así para el funcionamiento de la vida en familia, normas que regulen el desempeño de tareas, ocupaciones y roles. Es además una mezcla de sentimientos, comportamientos y expectativas, que impacta a cada miembro de la familia, lo cual permite que cada uno de ellos pueda desarrollarse como individuo. Por supuesto, dicha dinámica infunde el sentimiento de no estar aislado y de poder contar con el apoyo de los demás (Sánchez, 1996).

Es importante mencionar que todas las familias, sean funcionales o no, pasan por periodos de crisis; aunque en ocasiones disfrutan de áreas competentes, en otros momentos presentan funciones desadaptativas. Hay que tener una prudencia extrema a la hora de poner la etiqueta de "familia disfuncional", puesto que ello suele tener un impacto en las personas a quienes se les asigna dicha atribución. De la misma manera, la construcción de una sana dinámica familiar, propicia el espacio privilegiado de aceptación y amor de los seres humanos; es de gran relevancia el que esta emoción sea la que predomine en el clima de las relaciones intrafamiliares. Las familias constituyen un claro ejemplo de relaciones solidarias y cooperativas, ya que son capaces de unir distintas visiones en pro de un objetivo común. Es decir, las familias, de manera solidaria y colaborativa, co-construyen una forma de enfrentar las dificultades diarias y de ver el mundo, negociando cuando es necesario llegar a acciones comunes, o respetando y aceptando que los otros tengan distintas formas de ver el mundo y sus circunstancias.

En este punto se puede afirmar que los tres referentes teórico-conceptuales antes reseñados tienen un carácter muy general, lo que impulsa el perfil analítico del estudio, pero no una aproximación interpretativa. Se advierte también que, en el contexto colombiano, el abordaje de la educación sexual, en medio de una diversidad familiar regional y urbana tan compleja, junto con las dimensiones generadas por el capitalismo en América Latina, introducen variables de difícil categorización. En este orden de ideas, la investigación se encaminó a analizar el desarrollo de las dimensiones y funciones de la sexualidad de los NNA, situa- 
ción que demanda cambios en el sistema familiar, debido a sus necesidades de desarrollo que incorporan la independencia y la autonomía. E igualmente construir sentido acerca del aporte de la familia en torno al abordaje de la ES de los estudiantes, pertenecientes a las instituciones educativas oficiales del municipio de Armenia.

\section{MATERIALES Y MÉTODOS Método de investigación}

Por el carácter cualitativo de la investigación, en el contexto local, la indagación se orientó hacia el reconocimiento de las conversaciones compartidas con las personas, la percepción de la lógica de sus discursos, y la comprensión de sus vivencias, sentimientos y valoraciones, en el sentido sugerido por Strauss y Corbin (citados por Puyana; León \& Palacio, 2013). Con respecto al enfoque narrativo se espera reivindicar el relato de las personas como fuente legítima en la construcción del conocimiento, facilitando además el entendimiento del punto de vista de quienes producen y viven sus realidades (Puyana; León \& Palacio, 2013), como aproximación a la realidad de las familias de los estudiantes de Armenia y su relación con el abordaje de la ES.

\section{Población y Muestra}

Para el estudio se seleccionaron veintinueve instituciones educativas oficiales de la ciudad de Armenia, es decir, la totalidad de este tipo de establecimientos; se tuvo en cuenta en este sentido el acercamiento que sostiene la Secretaría de Educación con dichos colegios, más la facilidad de acceso a las familias de los estudiantes, por parte del equipo de investigación. En consideración a que la comunidad educativa, en las instituciones educativas, no se involucra de la misma forma en el abordaje de la educación sexual, se tomó como criterio de inclusión para la selección de los participantes, a aquellas personas que de forma directa o indirecta participan en dichos procesos escolares. Por tal razón se escogió a los docentes de las diferentes áreas, relacionados con el Proyecto de Educación para la Sexualidad y Construcción de Ciudadanía PESCC; a los funcionarios encargados de coordinar el PESCC o en su defecto a los orientadores escolares; así también a los padres, madres o acudientes de los estudiantes quienes a su vez, estaban registrados en el sistema de matriculados (SIMAT). Al tener un carácter intencional, la selección de los participantes y los datos presentados por ellos, no fueron generalizados en términos estadísticos, procedimiento que permitió respetar los aspectos bioéticos de la investigación.

\section{Técnica de recolección de los datos}

Las técnicas utilizadas consistieron en la entrevista semiestructurada, por un lado, la cual fue desarrollada a partir de un formulario que permitió recoger por escrito la información aportada por los docentes de educación física, los coordinadores del PESCC y los orientadores escolares. Por otro lado, la entrevista a profundidad, en donde se utilizó un guión para registrar en audio la información aportada por los padres, las madres y los acudientes. Dichas técnicas permitieron un autodiligenciamento por parte de los participantes, para el caso de la entrevista semiestructurada, así como la grabación en audio para la entrevista a profundidad; por supuesto, previo el consentimiento informado en ambos casos.

Una vez transcritas las entrevistas, se conjugaron dos procedimientos de análisis: el intratextual y el intertextual. El primero, se realizó a partir de una lectura detallada de cada una, con base en una matriz de categorización, teniendo en cuenta la trayectoria de los investigadores $\mathrm{y}$, en cuanto a los integrantes de las familias, se inspeccionó el significado explícito e implícito de las narraciones; tal procedimiento arrojó información novedosa sobre el tema, hasta encontrar la saturación de la información. De otra parte, con el análisis intertextual se obtuvieron comparaciones entre las diferentes narraciones, a partir de un minucioso proceso de codificación. Se elaboraron también las matrices a partir de las categorías analíticas establecidas por la aplicación estadística para las Ciencias Sociales, SPSS (IBM, 1989, 2010); y se agruparon los datos alrededor de los códigos sistematizados, con el soporte del Software Atlas Ti (Hernández; Fernández \& Baptista, 2010). Estas herramientas computarizadas permitieron mayor nivel de mayor elaboración para organizar los datos.

Al considerar el asunto de la codificación, como un proceso inicial de análisis se formularon unas primeras hipótesis de trabajo, se identificaron algunas categorías emergentes y a través de un análisis más profundo (principal) se procedió a realizar comparaciones de los relatos según cada entrevista, lo cual fue posteriormente triangulado con la teoría y los propósitos fundamentales de la investigación generando un proceso de conceptualización que evidencia el abordaje de la educación sexual al interior de las familias y su relación con la dinámica familiar. 


\section{RESULTADOS Y DISCUSIÓN}

Los resultados de la investigación sobre el Abordaje de la educación sexual de los profesores de diferentes áreas en las instituciones educativas de básica y media oficiales del municipio de Armenia, Quindío, expresan, en primer lugar, la descripción acerca de la información general sobre los profesores y funcionarios entrevistados, con respecto a su formación académica y los cargos desempeñados. Luego, en lo concerniente al Proceso de articulación del abordaje de la educación sexual en las instituciones educativas y las familias de los estudiantes de básica y media oficiales del municipio de Armenia, Quindío, se describió la profesión y el cargo de los participantes, se identificaron a los actores situados en las diferentes IE y se complementó revisando los proyectos, programas o actividades en los que participaron las familias. Finalmente, se analizó el Abordaje de la educación sexual y su influencia en la dinámica familiar, a partir de la descripción de las características sociodemográficas de los integrantes de las familias y la identificación de las variaciones en la dinámica familiar, teniendo como referente las vivencias y prácticas, visualizadas desde las dimensiones (Romero, 2001) y funciones de la sexualidad (Marina, 2005) de los NNA, en la perspectiva de la representación de la autoridad (Satir, 1984), la comunicación (Satir,1984) y las relaciones afectivas (Sánchez, 1996).

\section{Aspectos de la educación sexual abordados por los profesores de diferentes áreas que aportan al desarrollo de la sexualidad, en las familias de los estudiantes.}

Se identificó que en su gran mayoría los participantes son educadores, poseedores de títulos profesionales como licenciados en educación, pedagogía, biología, español y normalistas; aunque se presentan otras formaciones profesionales como psicólogos, gerontólogos y enfermeras. Estos actores representaron a las Instituciones Educativas Comfenalco, Zuldemayda, CASD, Oficial, Camilo Torres, Bosques de Pinares, Ciudadela del Sur, Ciudadela de Occidente, Teresita Montes, Las Colinas, ITI, Cristóbal Colon, Rufino José Cuervo Centro; y con menor representación, las instituciones Cámara Junior, INEM, Gustavo Matamoros, Eudoro Granada, El Caimo, Ciudad Dorada, Campestre y El Descanso de Mamá.

El cargo actual que desempeñan los educadores se concentra en las áreas de educación física, coordinadores de preescolar y orientadores escolares. De esta forma, el cumplimiento de las funciones o actividades propias del Proyecto Pedagógico de Educación para la Sexualidad se desarrolla en las mismas instituciones educativas, en los servicios de extensión a la comunidad, en las Organizaciones No Gubernamentales, o en el propio núcleo familiar. Se precisó además que las temáticas abordadas en el proyecto pedagógico de las instituciones educativas han tenido un mayor énfasis en los aspectos biológicos, los derechos sexuales y reproductivos, las competencias ciudadanas y la sexualidad; y en menor proporción, temas relativos a la educación sexual y la pedagogía. Las acciones, estrategias y actividades llevadas a cabo para el desarrollo de las temáticas mencionadas, tienen que ver con seminarios, cursos, encuentros, talleres y capacitaciones. Ocho instituciones no llevaron a cabo acciones de este tipo, y en consecuencia los docentes no respondieron.

Los principales resultados relacionados con la implementación de las acciones para el cumplimiento de las funciones del PESCC, según lo expresado por los profesores de educación física, tienen que ver con los cambios en los comportamientos y actitudes de los estudiantes. Otros argumentos aportados por los entrevistados afirman que algunos cambios fueron negativos durante las intervenciones del proceso, ya que no fueron valoradas por los estudiantes, ni se motivaron a participar. Se hallaron como principales dificultades la falta de motivación propia, la falta de recursos, la falta de capacitación y la falta de tiempo.

Los aportes profesionales para fortalecer dichas acciones son en su gran mayoría la realización de proyectos escolares, seguido de la producción de conocimiento, a partir de resultados de investigaciones acerca del tema. La disposición para el trabajo en su área disciplinar específica es su mayor dedicación, y en menor grado, la enseñanza de la religión, la ética y la moral. En cuanto a las estrategias para la vinculación de la familia en el proceso de la educación sexual, estas enfatizan en comunicados a padres, madres o acudientes, comentarios en las reuniones o talleres de padres de familia, las invitaciones a reuniones y la información a través de los mismos estudiantes.

El proceso de articulación del abordaje de la educación sexual en las instituciones educativas y las familias de los estudiantes de básica y media oficiales del municipio de Armenia, Quindío. 
Los participantes que correspondieron a los coordinadores del PESCC y los orientadores escolares pertenecían a las instituciones educativas Camilo Torres, Ciudadela de Occidente, INEM, Ciudadela del Sur, ITI, Bosques de Pinares, Teresita Montes, Las Colinas, CASD, República de Francia, Gustavo Matamoros, Cristóbal Colón, Escuela Normal Superior, Ciudad Dorada, Los Quindos, La Adiela, Ciudadela Cuyabra, Rufino José Cuervo-Sur, Nuestra Señora de Belén y Jesús María Ocampo. Fue importante para esta fase de la interpretación conocer la profesión de los participantes, dentro de las cuales se subraya que son educadores, licenciados en pedagogía o biología, psicólogos, pedagogos reeducativos; al igual que fue relevante reconocer el cargo que desempeñaban, correspondiendo estos a docentes, orientadores, coordinadores del PESCC y otros cargos.

Al preguntar acerca de si en la institución se cuenta o se han realizado proyectos, programas o actividades con las familias, referidas a la educación sexual, algunos respondieron negativamente, por encontrarse en una fase preparatoria. Se preguntó también sobre si en algún momento las realizaron y que narraran cómo fue la experiencia, pero sobre el particular no respondieron. Así mismo, al indagar acerca de la forma en que algunas entidades e instituciones que tradicionalmente acompañan los procesos de trabajo con las familias de los estudiantes, como la Secretaría de Educación Municipal, el grupo de investigación o la Universidad del Quindío, los participantes respondieron que con el desarrollo de talleres a los docentes.

A los participantes que respondieron que al interior de la institución se han realizado proyectos, programas o actividades con las familias, se les solicitó ampliar detalles sobre dichos eventos, y ellos precisaron que se desarrolla a través de la Escuela de Padres, mediante la realización de talleres, proyectos de investigación, capacitaciones y reflexiones en torno al contenido del manual de convivencia; el cual aborda de forma directa e indirecta aspectos relacionados con componentes o dimensiones de la sexualidad. Un elemento fundamental sobre la efectividad del proceso de gestión de los proyectos, programas o actividades, es contar con un plan de trabajo, aspecto que no fue mencionado por la mayoría. Algunos cuentan con un cronograma trimestral mientras que otros elaboran un calendario único de programación semanal o mensual.

Ahora, en relación con el proceso histórico de los proyectos, programas o actividades con las familias, algunos de los participantes lo relacionaron con las actividades de las Escuelas de padres, otros como un componente del PESCC, o un elementos asociado al PEI; otros en cambio, lo articularon al proceso del manual de convivencia o con otros proyectos institucionales. Respecto a quienes participaron en los proyectos, programas o actividades con las familias, los entrevistados respondieron que la comunidad en general, los docentes y los padres de familia.

En cuanto a los resultados obtenidos con el desarrollo de los proyectos, programas o actividades con las familias, se encontró que se han evidenciado algunos cambios positivos, constatados a partir del mejoramiento del dialogo con los hijos y el establecimiento de normas claras en el hogar, o fuera de él, entre otros. Otros manifestaron que aún no han valorado dicho proceso y los demás percibieron cambios negativos como la poca participación, lo cual es clasificado por los participantes como una situación limitante. Al preguntar acerca de un plan a futuro, los participantes manifestaron que se debe realizar algún tipo de seguimiento y que deben seguir haciendo parte del proyecto.

\section{La educación sexual y su influencia en la dinámica familiar.}

En relación con los miembros de las familias participantes en la investigación, el promedio de edad de los entrevistados fue de 40 años, con estudios en secundaria. Son oriundos de los departamentos de Caldas, Valle del Cauca y Putumayo, con una permanencia promedio de 4 años en la ciudad y la mayoría se desempeña como empleados. Un alto número son mujeres, quienes tienen el rol de madres, tías o acudientes de los NNA de las instituciones educativas oficiales de la ciudad de Armenia. Debido a estas funciones familiares, cuando se menciona el cuidado de hijos e hijas, sobrinos o sobrinas, de inmediato se reproduce la tradicional división del trabajo de esposa, ama de casa y acudiente institucional que se le asigna a "lo femenino", como visibilización del "contrato conyugal" (Fernández, 1993), predominante en las familias con características tipológicas nucleares completas e incluso en las reconstituidas, ya que el padre tiene como responsabilidad fundamental, el soporte económico de la familia. Cabe anotar que los estudiantes referenciados en la investigación pertenecen a las instituciones educativas INEM, Eudoro Granada, Ciudadela de Occidente, Rufino José Cuervo Sur, Bosques de 
Pinares, Luis Carlos Galán, La Adiela y Rufino José Cuervo Centro, de la ciudad de Armenia..

Los resultados de esta investigación coinciden, en cuanto a los aspectos anotados, con los obtenidos en la investigación titulada Familias colombianas y migración internacional: entre la distancia y la proximidad, en la que las autoras afirman que "en algunas de ellas que conforman hogares monoparentales es explicable culturalmente, que ante la separación o el divorcio, el padre renuncia a las funciones de cuidado, mientras que la madre asume el apoyo diario de hijos e hijas (Puyana; León \& Palacio, 2013).

De otra parte, al indagar acerca de los aspectos fundamentales que caracterizan la vivencia y la práctica de educación sexual, como insumos que aportan al desarrollo de la sexualidad en los integrantes de las familias de los estudiantes, se encontró que la forma en que se realiza la educación sexual en la actualidad corresponde a un proceso que se establece al interior de las familias, desde su propia historia vital. Es decir, desde los aportes que recibieron los padres, madres y acudientes en sus familias de origen, hecho que demarca, de una forma $u$ otra, las apuestas que realizan para su núcleo de convivencia. Una aproximación que permite explicar dicho entramado lo constituyen las expresiones de los participantes como el que se presenta a continuación:

“... lo único que le decían era que cuidado con los hombres, que lo embarazaban, siempre pensaban era en un embarazo, y que eso no podía ser para la familia sino eran casados" (entrevistado 001). “...No Realmente no, yo sé que de pronto en algún momento uno les preguntaba y ellos se incomodaban porque de pronto no tenían la manera adecuada de explicarle a uno, entonces en ese aspecto no, ósea, por eso como le digo lo que aprendimos en el colegio y lo que le contaban a uno los compañeros o lo que uno de pronto cogía un libro por ahí y podía pillar pero de resto no... simplemente iban sucediendo, como le digo o lo que escuchaba uno en el colegio pero a nosotros no, mi mama nunca nos explicó nada de eso" (entrevistado 007). "...te voy a decir que eso lo hice por inocente... pero nunca me habían explicado nada, ni uno que tiene que hacer, ni que es, ni nada, mi cuñada fue la que me oriento..." (entrevistado 008). “...el embarazo no fue planeado y cayó mal para todos fue frustrante, pues hablándolo desde la poca experiencia que tenía uno de la sexualidad...yo tenía 21 años y ella 17, la poca experiencia sexual por que incluso yo perdí la virginidad a los 21 años, yo no tenía ni idea que era la sexualidad, tal vez sabía que existían los condones y todo eso pero yo no sabía no me cuide, igual es que nunca tuve educación sexual, y la compañera de ese momento tampoco..." (entrevistado 012). “... ellos nos enseñaron a respetar a ser trabajadores, ninguno de nosotros salió malo, todos somos derechitos, de lo otro como planificar y eso no nos dijeron nada..." (entrevistado 013). “... pero lo único fue que nunca nos enseñaron con lo que uno iba a enfermar eso a mí nunca me lo enseñaron y cuando yo llegue el día que fui a enfermar entonces yo dije mami mire que me está saliendo algo y yo no sé si fue que me chusé, entonces mi hermano el mayor dijo que le salió y yo pues me manché los calzones y dijo de qué color, y no me dijo como me la colocaba si no que yo me la coloque al contrario (risas), si me entiende" (entrevistado 014).

De lo anterior se colige que con la manifestación de algunos rasgos, acciones, actitudes y experiencias se caracterizan las consecuencias del abordaje de la educación sexual, a partir un proceso autoritario, machista y desde la pedagogía del silencio. Este entorno dramático fue recibido-asumido en la convivencia con la familia de origen, si nos ajustamos a la narrativa; en donde se plasma además, una significativa partida del hogar a temprana edad, relacionada directamente con el aburrimiento o la violencia doméstica y el desconocimiento de aspectos básicos acerca de la educación sexual.

Por consiguiente, las variaciones en la dinámica de las familias de origen, desde la representación de la autoridad, se perpetuaban a través del establecimiento de reglas o normas de carácter rígido e impositivo por parte del padre hacia los hijos; lo que incluye que en ocasiones el mismo padre no cumpla con sus propias reglas. Mientras la madre, de estirpe cuidadora y protectora, tiene como misión la supervisión a través del cumplimiento de los roles propios de su género; ello se puede evidenciar así:

“... Siempre nos trataron bien, pero no nos consentía mucho, nos pegaban con ramales... aunque a mí no tanto no porque yo era como achapada a mi hermana sino ella si tenía cositas como maluquitas, era como uñonsita (Ladrona)...”(entrevistado 002). “... yo vivía con mi mama, con mis tías, toda la familia y pues vivíamos bueno. Mi papa se murió en un ac- 
cidente; por parte de mama tengo una hermana y por parte de papa tres... siempre nos trataron bien, pero no nos consentía mucho, nos pegaban con ramales... "(entrevistado 003). “... tranquila gracias a Dios, fue muy estricta de la casa al colegio y del colegio a la casa, no le gustaban los trabajos en grupo, ella se dedicó a trabajar y mi abuelita nos crio, se encargaba de nosotros, hasta que falleció y ahí ya mama se dedicó al trabajo en la casa peluquería, cosas en la casa, y mama nos daba ejemplos, nos mostraba espejos de las demás personas de no ir a cometer errores, pero como uno siempre..."(entrevistado 004). “... en la casa le enseñan a uno muchos valores a uno, el respeto por los demás, ser honrado, sincero en lo que dice y hace, constante, ser muy hogareño, porque eso no lo inculcaron mucho en la casa el estar siempre pendiente de la casa y los hijos... y salir siempre adelante con una meta...”(entrevistado 005). “... muy estrictos, pero hoy veo que eso sirvió, nos regañaban nos pegaban, trabajábamos todos por parejo, íbamos a la escuela, mi papa muy estricto, donde no se podía responder así uno tuviera la razón, charlar sí, pero con mucho respeto, no se podía contradecir a los padres... "(entrevistado 010).

Respecto a la comunicación, había poco diálogo con los hijos, ya que la formación se basaba en la experiencia por fuera del hogar, entorno abandonado a temprana edad, sobre todo por parte de los hombres. Estos testimonios ratifican esa vivencia:

“... es que yo siempre estudiando, nos daban estudio, pero la expectativa de él era que uno saliera casado de la casa o sino no podía volver a la casa, esas palabras siempre él las decía. Somos 11 hermanos, yo soy de las últimas, mandaba papa, siempre vivimos en una finca. Mama se dedicaba a la casa, al hogar, nos criaba, se encargaba de levantarnos, a alimentarnos, nos enseñaban a trabajar, a comportarse bien, a ser gente servicial. Maltrato sí, no siempre pero si, de palabras, de hechos. No podía hablar tranquilamente con los papas no se podía hablar con ellos..." (entrevistado 001). “... la que daba la parte disciplinaria era mi mama, ella era muy verbal, alegaba y alegaba, y pues la chancla a mí me tocó la época de la chancla, que ella lo regañaba a uno y tenga su chancletazo, mi papa creo que en la vida solo me dio una pela, que yo recuerde...” (entrevistado 009). “... muy estrictos, pero hoy veo que eso sirvió, nos regañaban nos pegaban, trabajábamos todos por parejo, íbamos a la escuela, mi papa muy estricto, donde no se podía responder así uno tuviera la razón, charlar sí, pero con mucho respeto, no se podía contradecir a los padres... ella pues cariñosa sí, pero ella le seguía la corriente a mi papa, aunque ella nos quisiera defender ella seguía las rutas de mi papa... entonces por eso yo dije vamos a empezar aportar para tener voz y voto y que mama no tenga que estar ahí con papa..."(entrevistado 010). “... Las normas en la casa eran para mí, porque al niño (mi hermano medio) no, yo tenía que llegar temprano, hacer las cosas de la casa, estudiar y si no lo hacía tenga la pela, el castigo, cambio el si podía hacer lo que quisiera que volear piedra, insultar la gente y a él sino le decían nada..." (entrevistado 012).

En cuanto a las relaciones afectivas, el vínculo emocional no era cercano, ni el tiempo para compartir los espacios familiares era permanente, mucho menos las relaciones familiares se caracterizaban por la disposición o la facilidad para expresar los sentimientos. Así lo experimentaron algunos de los participantes:

“...mi infancia, mi madre se casó muy joven también, vivió en Cali tuvo 3 niños también, que eran mi hermana, mi hermano que fue asesinado a los 18 años y mi persona, entonces hubo muchos problemas familiares y se separaron... mi madre nos llevó muy joven al putumayo, vivía mis abuelos allá por parte de mi mama, entonces se radico allá y pues siempre nos criamos mi abuela, mis hermanitos y una prima, después asesinaron a mi hermano..." (entrevistado 004). "...mi mama me llevo a vivir con mi papa desde los 6 años y me crie con el cómo hasta los 12 años, sufrí mucho por que consiguió otra señora que nos maltrataba, nos daba garrote todo el que quiera... pues mi mama me decía que se separó porque mi papa le daba muy mala vida, que la maltrataba, le pegaba, pues yo estaba muy pequeñita y no me recuerdo, pero eso es lo que cuenta ella, y en si pues familia no hemos tenido..."(entrevistado 005). "...mi historia es muy triste, yo me crie con los abuelos, ya después de los 12 años me fui a vivir con mi mama, por allá en una finca y no me dio estudio... mi papa y mi mama se dejaron desde que yo tenía 3 meses de nacida, entonces nosotros casi no pasamos con mi papa, entonces yo vine a distinguir a mi papa cuando yo tenía como 13 años... "(entrevistado 006). “...yo soy de una familia numerosa 14 hermanos, la segunda de arriba hacia abajo, me toco criar la mayoría de mis hermanos, cuando tenía como 15 años vi la necesidad de salir y aportar económicamente a la casa, salí de allí en contra de mi papa y con consentimiento de mi mama, me fui para 
donde mi abuela allá trabaje en un almacén luego en una fábrica que quedaba cerca, muy contenta porque ya colaboraba para mi casa, para mis 10 hermanos... "(entrevistado 010). “...no pues la vida mía fue que mi mama nos crio, porque pues según eso cuando yo nací, tenía un mes de nacida cuando mi papa dejo a mi mama, nosotros somos de una familia grande nosotros éramos 18 y mi mama dice por descuido de mi papa se murieron 9...”(entrevistado 011). “... bueno yo vengo de una familia donde desafortunadamente yo no me crie con mi papa, porque mi mama se casó con un hombre de más de 40 años... con el pasar del tiempo yo me di cuenta que el señor con que vivía mi mama no era mi papa... yo soy la única hija del matrimonio, los otros dos son del papa con el que me crie..." (entrevistado 012).

Para el caso de las dimensiones de la sexualidad, la aproximación biológica se relaciona con el cuidado del cuerpo, los cambios morfo-fisiológicos, la salud, las diferencias según las características sexuales secundarias, entre otros. En relación con la dimensión socio cultural, emerge la falta de orientación, frente al establecimiento de normas y roles reconocidas, de acuerdo con las características particulares de los grupos de convivencia. Así mismo, desde la dimensión psicológica, se percibe un nulo proceso de auto reconocimiento afectivo-cognitivo, acompañado de poca adquisición de procesos de autoestima y toma de decisiones. Y en cuanto a la dimensión ética-filosófica, se enfatiza la determinación de procesos valorativos, desde la imposición y la violencia, muchas veces física.

En este sentido, en cuanto a las funciones de la sexualidad, hay testimonios que corroboran la dimensión biológica, como aquí se expresa: “... estamos hablando es del pasado de cuando yo tuve la relación; ahí fue cuando yo quede embarazada del hijo mío... del mayor y entonces yo ese día como que me di cuenta, pues yo un susto porque yo no planificaba, no sabía de eso, no sabía de educación sexual..." (entrevistado 001) “...el embarazo no fue planeado y cayó mal para todos fue frustrante, pues hablándolo desde la poca experiencia que tenía uno de la sexualidad... que no existe una orientación familiar frente a los procesos de la reproducción, ni de métodos de planificación familiar..." (entrevistado 012).

En cuanto a las demás funciones, se resalta que no se menciona la función erótica, aunque aparecen algunas manifestaciones tácitas desde lo prohibido por la religión, tal como se evoca. Así mismo, sobre la función afectiva, no se considera importante saber que el otro siente, sobre lo cual hay un claro entendimiento. Por lo contrario se valora que el compañero cuenta con recursos para responder en el hogar. Con respecto a la dimensión comunicativa-relacional, las familias no acompañan la forma, ni las estrategias en que se realiza un adecuado establecimiento de métodos comunicativos con los otros.

Con base en lo anterior, se evidencia que el abordaje de la educación sexual desde la familia de origen tiene como resultado el abandono del hogar a temprana edad, el establecimiento de la unión conyugal consensuada o no, el nacimiento de hijos planeados o no, Adicionalmente se refleja la carencia de roles, la ausencia del padre, la presencia de violencia de pareja y familiar, el poco establecimiento de normas y límites entre los integrantes de la familia, hecho que debilita las reglas de autoridad. Es sintomático la rigidez en las relaciones intrafamiliares, en donde se asume el rol materno como impositivo y con muchas dificultades, circunstancias que son narradas por los participantes como lo característico de sus historias de vida.

En cuanto al abordaje de la educación sexual en la familia de convivencia, se encontró de forma reiterativa que aún perduran procesos inherentes a la pedagogía del silencio, con contenidos irrelevantes que no interesan en la actualidad. Por tanto, los mecanismos de comunicación son poco efectivos y afectivos entre sus integrantes, dado que el esfuerzo no sobrepasa la intención de conformar una relación de pareja estable, concomitante con el control natal y la prevención de infecciones de trasmisión sexual; en tal contexto pasan a un segundo plano el desarrollo personal, académico, profesional, laboral y social de los hijos.

Respecto a las familias de convivencia, los hallazgos distan de lo encontrado en las familias de origen, que establecen un cumplimento de las propias reglas por parte de padres y madres, cuya enseñanza de las normas y reglas al interior del hogar son compartidas y consensuadas con la pareja y demás integrantes de la familia. En este sentido se dejan de lado el castigo físico como correctivo de los NNA y se abren escenarios en donde se fortalece el diálogo, mediante el uso de un lenguaje común con adecuados canales de comunicación. Así pues, se permite el establecimien- 
to de relaciones de confianza con los hijos, aunque en la formación para la vida, sigue primando la prevención del embarazo y de las Infecciones de Trasmisión Sexual ITS; acompañado ello de la exigencia sobre la conformación de una pareja estable, aspectos que no alcanzan a abarcar de forma amplia las dimensiones y las funciones de la sexualidad en su totalidad.

A continuación se presentan algunos de los testimonios brindados por los participantes que permiten vislumbrar estos hallazgos:

“...en cuanto a educacion sexual se les han enseñado todo lo de proteccion, para no quedar embarazadas, todos los metodos ellos los conocen, y ahora en este momento el niño esta recibiendo educacion sexual en el colegio...pero de parte de nosotros yo trato de metermele pero el dice que le da pena...no hay confianza para eso, yo le digo cosas de niñas y todo y el dice que todavia no, que gas... más bien hablamos de lo mucho que lo adoro, de lo importante que es para mi, pero de otras cosas no... de mujeres o sexualidad no..."(entrevistado 01). “...ósea el niño de 15 años es muy abierto, y él ahora tiene una noviecita y me dice que hasta el momento nada (risa)...y yo si le digo cuentecita mijo, si algo cuenta para comprarle condones y él se ríe, me dice no mama yo a ella la respeto y ya llevan como año y medio, y estudian pero la niña es como cristiana o evangélica..." (entrevistado 06). “... la verdad nuestra relación como padres ha sido muy extrovertida con la niña, yo hasta los 7 años me bañe con ella nos metíamos al baño desnudos, yo la estregaba ella me estregaba, hasta que llegó el momento en que ella preguntaba esto para que es... entonces yo le iba contando, porque pues si yo no sabía eso de la casa la vida me había enseñado afrontar ciertos temas, la sexualidad, lo que es el cuerpo, abordándolo desde muchos puntos de vista, más de lo que es la persona, el alma, el cuerpo y de ahí vamos rellenando con el resto de temas... de lo otro pues nosotros siempre le decimos que la sexualidad es responsable que ella es libre de ejércela, todo eso... el proyecto de vida, las metas que tiene para realizar...incluso ella ya tuvo relaciones y pues ella me conto a mí y yo le dije que se cuide mucho, que no solo con condones sino con respeto al cuerpo..." (entrevistado 09). “...tengo dos hijos, el niño va cumplir 20 años y la niña 18 años, mírelos... yo he tratado de no ser la madre más liberada pero no tampoco la más apretada, les enseñe el respeto por las personas, los abuelos, los gay, los paralíticos, siempre he tratado que ellos vean muy normales esas situaciones, porque no me gusta que ellos discriminen y todo eso... yo he tratado que ellos me tengan confianza, que me cuenten todo... Mi hijo perdió la virginidad casi a los 18 años, y el médico le dijo que si era que él nunca había estado con una mujer, entonces yo he tratado de que la vida sexual de ellos sea muy de confianza, yo los siento y les digo que se deben cuidar utilizar la planificación y todo, porque ellos están jóvenes y pues primero está el proyecto de vida y los estudios...todo se puede negociar, menos que le pongan la casa de motel a uno (Risa)..." (entrevistado 012).

No obstante, se da una mayor importancia a la formación en valores como el respeto, el amor y la responsabilidad, lo que genera apertura y asertividad en la comunicación. Los vínculos familiares por su parte, se fortalecen debido a que comparten el tiempo libre, las expresiones de afecto y el apoyo familiar, éste último utilizado como un espacio para llegar a acuerdos, solucionar problemas y asumir responsabilidades en momentos de crisis. De igual forma, en cuanto al aporte de la familia para la construcción de sentido sobre una sana educación sexual, los actores manifestaron que la unión familiar es el espacio privilegiado para acompañar el proceso educativo de las instituciones. Indican que ello será posible, por supuesto, a través del afecto y la confianza entre sus integrantes y teniendo gran relevancia el que esta emoción sea la que predomine en el clima de las relaciones intrafamiliares; espacio donde sea posible compartir tiempo, fortalecer valores, negociar normas de convivencia y aceptar que los otros tengan distintas formas de ver la sexualidad.

\section{CONCLUSIONES}

Las estrategias para la ES en las instituciones de educación básica y media oficiales de Armenia se resumen en actividades de información, comunicación y educación continua. Es claro que el apoyo interinstitucional está cobrando mayor interés para las familias, como aquel espacio socializador secundario, desde donde se validan y realimentan la cultura y los valores. Este abordaje se hace desde el ámbito de la Promoción y la Prevención en los NNA que se encuentran en las edades del preescolar y escolar, como también a través del componente de Salud Sexual y Reproductiva en los adolescentes, los cuales adquieren las bases de su comportamiento y conocimientos, su sentido de responsabilidad y la capacidad de observar, pensar y actuar. 
El proceso de articulación se está haciendo a través de la estrategia de Escuelas de Padres, en donde se propicia la realización de talleres, proyectos de investigación, actividades de capacitación y reflexiones sobre el contenido del manual de convivencia. Esto conlleva a involucrar en sus acciones a las familias, respetando sus nuevos arreglos y funciones en las que hay separación de los ámbitos de la sexualidad, la gestación, el matrimonio, la crianza y las relaciones familiares, para mayor entendimiento de las relaciones de género y de crianza que rehacen las familias desde otros enfoques y prácticas.

Es relevante en esta investigación que las características sociodemográficas de las familias (alfabetas, mayores de edad, empleados, con diversas tipologías familiares, y estabilidad habitacional, entre otras) no afectan de forma relevante el abordaje de la educación sexual, la cual está centrada básicamente en los diferentes contextos de la misma forma a orientar a los hijos e hijas hacia la consolidación de parejas estables, la planificación del número de hijos y la prevención de infecciones de transmisión sexual.

En lo relacionado con la autoridad, la comunicación y las relaciones afectivas, categorías analíticas que corresponden a la dinámica familiar, se encontró que estas presentan diferencias notorias en cuanto a la familia de origen (normas y reglas de carácter rígido e impositivo, centrado en el padre; poco diálogo con los hijos, vínculos emocionales poco cercanos, falta de tiempo compartido, y baja expresión afectiva) y la familia de convivencia (reglas compartidas y consensuadas, adecuados canales de comunicación, establecimiento de relaciones de confianza y relaciones afectivas al compartir tiempo, expresar afecto y sentimiento de apoyo familiar); aspectos estos que no modifican radicalmente el proceso de educación sexual, manteniéndose al interior de las familias la necesidad de solicitar a los hijos alejarse de parejas inestables, evitar embarazos no deseados y no exponerse a las infecciones de transmisión sexual; se incorporan como aspectos novedosos en la ES la formación en valores basada en el respeto y la responsabilidad.

El aporte más significativo de la familia, para la construcción de una sana educación sexual, se refiere al acompañamiento permanente del proceso educativo y la participación en las Escuelas de Padres convocadas por las instituciones, fortaleciendo al mismo tiempo las relaciones intrafamiliares mediante las expresiones de afecto y la confianza entre sus integrantes: compartiendo tiempo, fortaleciendo valores, negociando normas de convivencia y aceptando que los otros tengan distintas formas de ver la sexualidad. 


\section{BIBLIOGRAFÍA}

- Arreguez, Ó. F. (2013). Factores que condicionan al personal de enfermería a brindar un abordaje educativo de calidad en salud sexual y reproductiva a las pacientes hospitalizadas por aborto. Mendoza, Argentina: Universidad Nacional de Córdoba.

- Cardinal De Martin, C. (1975). Apuntes sobre sexo, sexualidad humana y educación sexual.Corporación Centro Regional de Población, Colombia.

- De La Rosa Bobadilla, J. J., Fernández Rincón, C. A., Giraldo, C., Forero Arango, L., Soto Rivas, M., Mondragón Sánchez, E. J., \& Fajardo Torres, K. L. (2010). Nuevas configuraciones de las familias en los hogares de los estudiantes de las instituciones educativas de básica y media oficiales del municipio de Armenia Quindío, 2010. Documentos de investigación. Universidad del Quindío, 1-43. Obtenido de http://repositorio.uniquindio.edu.co/bitstream/123456789/121/1/Nuevas\%20 configuraciones $\% 20 \mathrm{de} \% 201$ as $\% 20$ familias $\% 20 \mathrm{en} \% 201$ os $\% 20$ hogares $\% 20 \mathrm{de} \% 201 \mathrm{los} \% 20$ estudiantes $\% 20$ de $\% 201$ as $\% 20$ instituciones $\% 20$ educativas $\% 20 \mathrm{de} \% 20 \mathrm{~b} \% \mathrm{C} 3 \% \mathrm{~A} 1$ sica $\% 20 \mathrm{y} \% 20$ media $\% 20$ oficiales\%20del\%20municipi

- Fernández Rincón, C. A., \& Lopez Cardozo, L. I. (diciembre de 2011). La educación sexual como tema transversal de las instituciones públicas educativas de la básica primaria en Armenia, Colombia. Revista Cultura del Cuidado, 8(2), 46-57.

- Fernández, A. M. (1993). La mujer de la ilusión. Pactos y contratos entre hombres y mujeres. Buenos Aires: Paidós.

- Hernández Sampieri, R., Fernández Collado, C., \& Baptista Lucio, M. d. (2010). Metodología de la Investigación (5 ed.). México: McGraw-Hill / Interamericana editores, S.A. de C.V.

- Marina, J. A. (2005). El rompecabezas de la sexualidad. España: Editorial Anagrama.

- Mineducación. (1993). Resolución número 3353 de 2 de julio de 1993. Por el cual se establece el desarrollo de programas y proyectos institucionales de Educación Sexual en la Educación básica del País. Bogotá: El Ministerio.

- Organización Panamericana de la Salud. (1995). Salud sexual y reproductiva. Washington: Organización Panamericana de la Salud.

- Puyana, Y.; León, A. \& Palacio, M. C. (2013). Familias colombianas y migración internacional: entre la distancia y la proximidad. Bogotá: Universidad Nacional de Colombia, Facultad de Ciencias Humanas.

- Romero, L. (2001). Elementos de la sexualidad y Educación sexual. Bogotá: Centro de asesoría y consultoría CAC, Bogotá.

- Rubio, E, (1994). Antología de la sexualidad humana. Conapo. México. Tomo I.

- Sánchez Salazar, R. D. (1996). Dinámica Familiar. Relaciones Intrafamiliares. Manizales: Editorial Universidad de Caldas.

- Satir, V. (1984). Relaciones humanas en el núcleo familiar. México: Editorial Paz-Mexico.

- Weeks, J. (1998). Sexualidad. México: Paidós Ibérica. 